\title{
Die Lungenkrankheit von Thomas Bernhard - Realität und Fiktion ${ }^{1}$
}

\author{
The Lung Disease of Thomas Bernhard - Reality and Fiction
}

Bibliografie

DOI http://dx.doi.org/ 10.1055/s-0029-1215364 Pneumologie 2010; 64: 111-114 @ Georg Thieme Verlag KG Stuttgart · New York ISSN 0934-8387

Korrespondenzadresse Prof. Dr. med. Ralf Wettengel Schillbachstraße 13 07743 Jena

r.wettengel@web.de
Falls ein erklärter Autobiograph seine persönlichen Themen in einer detaillierten Erzählung verarbeitete, deren Realität sich von seiner eigenen Geschichte deutlich unterschiede ... und einer Reihe von Ereignissen Bedeutung verliehe, die nie stattgefunden haben, wären wir nicht gerade überrascht, wenn man ihm vorwürfe, dass die Darstellung seines vermeintlich wahren Lebens eine ausgesprochene Lüge sei.

Philip Roth [1]

\section{Zur Perzeption der Lungenkrankheit von Thomas Bernhard \\ $\nabla$}

Anlässlich seines 15-ten Todestages wurde in Weimar die Thomas-Bernhard-Nacht veranstaltet. Die Thüringer Landeszeitung stellte den Schriftsteller ihren Lesern vor als den „zeitlebens Stigmatisierten, Gequälten, unter einer durch grässlichste Kunstfehler von inkompetentesten Ärzten verschlimmerten, wenn nicht sogar hervorgerufenen Lungenkrankheit Dahinsiechenden.“

Für Elfriede Jelinek erklärt die Lungenkrankheit den charakteristischen Stil dieses Autors. Sie schreibt in einem Nachruf:

„An diesem toten Giganten wird niemand mehr vorbeikommen. Seine lebenslange Krankheit hat ihn herausgehoben, seinen stets fehlenden Atem hat er festschreiben müssen. Daher war seine Literatur eine Literatur des Sprechens ... der Endlostirade. Solange ich spreche, bin ich. Auch wenn man längst kannte, was da gesagt wurde ... musste man, selbst atemlos geworden, immer weiterlesen. So hat die Erfahrung des zu wenig Luftkriegens den wüsten Atem des Sprechens erzeugt.“ Manfred Mittermayer, Philologe, promoviert mit einer Arbeit zu Thomas Bernhard, hat in seinem Festvortrag aus Anlass des 40-sten Kongresses der DGP und des 25-sten Kongresses der Österreichischen Gesellschaft für Lungenerkrankungen

${ }^{1}$ Helmut Fabel zum 76-sten Geburtstag gewidmet.

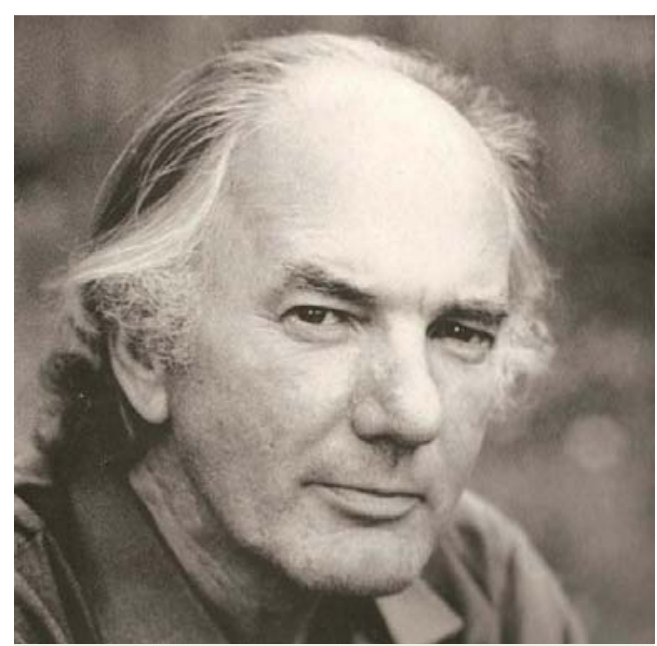

Abb. 1 Thomas Bernhard.

und Tuberkulose über „Thomas Bernhard und die Lungenkrankheit - Beobachtungen an seinen autobiographischen Schriften“ referiert [2]. Er interpretiert die berühmte Szene im Badezimmer, das vom Autor als Sterbezimmer bezeichnet wird, als den „nachdrücklich als eigenständig reklamierten Entschluss, die lebensgefährliche Krankheit zu überwinden, den Tod im Rahmen eines bewusst gesetzten Willensaktes zu besiegen... als Ergebnis individueller Selbstermächtigung."

In der autobiografischen Erzählung „Der Atem eine Entscheidung“ liest sich das so:

„Plötzlich hat der Atem des Mannes vor mir aufgehört. Ich will nicht sterben, denke ich. Jetzt nicht ... Ich wollte leben ... und zwar mein Leben, wie und solange ich es will ... Das hatte sich der, der schon aufgegeben gewesen war, in dem Augenblick, in welchem der andere aufgehört hatte zu atmen, vorgenommen. Von zwei möglichen Wegen hatte ich mich in dieser Nacht in dem entscheidenden Augenblick für den des Lebens entschieden ..." [3]. 
Offensichtlich wird die Schilderung dieser Krankheitsphase, in der „der ohnmächtig Daliegende seine Existenz wieder in die Hand nimmt“, so Mittermayer, als Tatsachenbericht verstanden. Die ungewöhnliche Situation, dass ein bewusstloser, von den Ärzten angeblich bereits aufgegebener Patient durch einen Willensakt, der ja ein klares Bewusstsein voraussetzt, den Tod besiegt, scheint dabei nicht zu stören.

Thomas Bernhard hat drei weitere autobiografische Erzählungen veröffentlicht [4-6]. Sorgfältige Nachforschungen von Huguet [7] haben ergeben, dass die geschilderten Personen und Lebensumstände mit der Realität nicht übereinstimmen. Deshalb urteilt Höller in seiner Bernhard-Biografie: „Die autobiographischen Erzählungen Thomas Bernhards sind fiktionale Texte. Man darf sie nicht an den Details der faktischen Wirklichkeit messen “ [8]. Dieses Eingeständnis überrascht, denn es widerspricht der Erwartung des Lesers einer Autobiografie ebenso wie der erklärten Absicht des Autors, die Ereignisse und Geschehnisse „mit dem Willen zu Wahrheit und Klarheit" zu notieren [3].

Allerdings ist Höller bezüglich des Wahrheitsgehalts der Krankengeschichte weniger kritisch. In dem Kapitel „Gegen den Tod schreiben“ heißt es:

„Die Ursache dieser Todeskrankheit lag in der schweren Lungentuberkulose, die sich Thomas Bernhard infolge der nassen Rippenfellentzündung 1949 zugezogen hatte. Die autobiografischen Erzählungen, besonders Der Atem und Die Kälte beschreiben die Geschichte dieser lebensgefährlichen Erkrankung."

War die Tuberkulose des Thomas Bernhard lebensgefährlich? Höller glaubte das, denn es gab die Sterbeszene und die suggestive Beschreibung der letzten Ölung. Er lobt den Realismus dieser Pathografie, betrachtet also einen Text, den er insgesamt als fiktiv bewertet, in medizinischer Hinsicht als Tatsachenbericht. Dazu verführt ihn die vermeintliche medizinische Versiertheit des Patienten. In der Tat erweckt die Art, wie sich der Autor in der Erzählung „Die Kälte“ als die Ärzte kontrollierender, insgeheim die Anweisungen erteilender Spezialist darstellt, den Eindruck von Expertenwissen. Davon wird später noch die Rede sein.

\section{Die Krankengeschichte \\ $\nabla$}

Der 17-jährige Thomas Bernhard erkrankt mit einer Pleuritis exsudativa und wird im Landeskrankenhaus Salzburg behandelt. Ausführlich wird eine Pleurapunktion geschildert. Das Durchstechen der Brustwand war nicht schmerzhaft, aber beim Anblick des Gummischlauchs und des Behälters - des gleichen roten Gummischlauchs und des Gurkenglases, die er als kaufmännischer Lehrling zum Essigabziehen verwendet hatte - , wird ihm übel, er kollabiert und wacht erst im Krankensaal wieder auf. Nach und nach kommt er wieder zu sich, aber er sieht alles nur verschwommen, „die Gegenstände waren undeutlich, schließlich überhaupt nicht mehr wahrnehmbar gewesen, die Stimmen hatten sich entfernt....mein Bett wird auf Räder gehoben...Ich bin im Badezimmer. Ich weiß, was das bedeutet" [3].

Was bedeutet es wirklich? Was geschieht? Die Schwester kommt ein paarmal herein, hebt die Hand auf, zählt den Puls, nebenan stirbt angeblich jemand, aber er hat es nicht gesehen, nur gehört, dass der Patient neben ihm aufgehört hat zu atmen, und gegen fünf Uhr früh kommen Pfleger und bringen ihn in den Krankensaal zurück. Merkwürdig ist nun: nicht im Badezimmer, dem angeblichen Sterbezimmer findet die letzte Ölung statt, eine sondern in der sicheren Umgebung des Krankensaals, und zwar exakt gegen sechs Uhr früh. Die Einzelheiten sind dem Erzähler wichtig: es liest sich wie ein Tatsachenbericht.

Aus ärztlicher Sicht handelt es sich bei einem Kreislaufkollaps nach einer Pleurapunktion um einen banalen Zwischenfall. In dem detailreichen Bericht über das Ereignis ist von medizinischen Maßnahmen, die auf eine protrahierte Kreislaufreaktion hinweisen würden, wie wiederholte Blutdruckmessungen, Anlegen einer Infusion etc. nicht die Rede. Selbst wenn man eine lebhafte Phantasie und die verständliche Ängstlichkeit des jungen Patienten berücksichtigt, wirkt die Rekonstruktion des Ereignisses aus der Sicht des etwa 50-jährigen Erzählers übertrieben:

„Ich wollte leben, alles andere bedeutet nichts...Das war kein Schwur, das hatte sich der, der schon aufgegeben war, in dem Augenblick, in dem der andere aufgehört hatte zu atmen, vorgenommen."

Die recht spekulative Überlebensbegründung scheint aber den Autor selbst nicht befriedigt zu haben, denn zwei Sätze weiter lesen wir: „Die Tatsache, dass die schwere nasse Wäsche nicht auf mein Gesicht gefallen war“ - im Badezimmer war Wäsche an einem Strick aufgehängt gewesen und heruntergefallen - „und mich nicht erstickt hatte, war die Ursache dafür gewesen, dass ich nicht aufhören wollte zu atmen.“

Wie sich bei genauerem Lesen zeigt, ist dieser für die Erzählung zentrale, den Titel Der Atem - eine Entscheidung begründende Text also nicht nur medizinisch unplausibel, sondern auch inkonsistent.

1949, ein Dreivierteljahr nach dem Aufenthalt im Landeskrankenhaus Salzburg, wird Thomas Bernhard in ein ehemaliges Hotel, das „Vötterl“ geschickt, das in der Nachkriegszeit als „Erholungsheim für an den Atemwegen Erkrankte“ diente. Wir zitieren die letzten Sätze der genannten Erzählung: „ Zwei Wochen nach meiner Entlassung aus Großgmain hatte mir die Krankenkasse einen sogenannten Einweisungsschein in die Lungenheilstätte Grafenhof zugeschickt. Mit der an diesen Einweisungsschein gehefteten Fahrkarte hatte ich meine Reise antreten können.“ Offenbar handelte es sich um eine Maßnahme der Tuberkulosefürsorge. Ein aktueller Behandlungsgrund bestand nicht.

Der erste Aufenthalt in der Lungenheilstätte Grafenhof dauerte sieben Monate (7/49-2/50). Das Krankenblatt enthält u.a. sorgfältig geführte Tabellen über sieben Temperaturmessungen pro Tag (alle Werte afebril), Eintragungen über bakteriologische Sputum-Untersuchungen (stets negativ) und die Beurteilung der Thoraxaufnahme: induriertes tuberkulöses Infiltrat rechts parahilär, Pleuraschwarte rechts. Ein Verlaufsbogen wurde nicht geführt. Im Januar 1950 erfolgte eine Streptomycin-Behandlung mit $2 \times 0,25 \mathrm{~g}$ über zehn Tage. Weitere Therapie: Lebertran, Einreibungen mit Salicyl-Spiritus, Freiluft-Liegekur. Der Patient wird als geheilt entlassen. ${ }^{2}$

Dies war leider ein Irrtum. Bei einer Nachuntersuchung zwei Monate später wird ein Infiltrat festgestellt. Vom 25.4.-6.7.1950 wird Thomas Bernhard erneut im Landeskrankenhaus Salzburg behandelt. Laut Arztbrief vom 12.7.50 hat es sich um ein frisch aufgetretenes Infiltrat im rechten Lungenunterlappen mit erbsgroßer Aufhellung gehandelt. Erstmals waren Tuberkelbakterien nachweisbar. Da eine Pneumothorax-Anlage wegen der Pleuraschwarte nicht möglich war, erfolgten eine Phrenicus-Quetschung und die Anlage eine Pneumperitoneums. Der Patient erhielt $20 \mathrm{~g}$ Streptomycin, kombiniert mit Tuberkulin. Die Sputumkonversion ist am 3. 7. 1950 dokumentiert.

\footnotetext{
2 Dr. Peter Fabian danke ich für freundlich gewährte Einsichtnahme in Krankenakten und bereitgestellte Röntgenaufnahmen.
} 
Zur Nachbehandlung wird Thomas Bernhard erneut in die Lungenheilstätte Grafenhof verlegt. Dieser zweite Aufenthalt dauerte sechs Monate (7/50-1/51.) Laut Krankenblatt war der Verlauf unauffällig, die Körpertemperatur stets afebril, BSG 2/4; bei zwei Larynxabstrichen kein Keimnachweis. Die Tuberkulose wurde offenbar als ausgeheilt betrachtet, eine weitere medikamentöse Behandlung nicht für erforderlich gehalten.

Auszug aus dem Krankenblatt:

Beurteilung der Thoraxaufnahme vom 2.1.1951: In der rechten Lunge ziemlich harte Streifenschatten vom Hilus nach basal. Zwerchfellhochstand rechts. Elf Nachfüllungen des Pneumoperitoneums mit jeweils $1000 \mathrm{ml}$. Wiederum existiert kein Verlaufsbogen. Ob ein Arzt mit dem sensiblen, phantasiebegabten jungen Mann jemals ein intensiveres Gespräch geführt hat, ist nicht zu eruieren. Der Patient hat die Ärzte bei den Visiten als „die vor dem Bett aufgestellte weiße Mauer, in der kein menschlicher Zug zu entdecken war“, erlebt oder sie zumindest im Rückblick so beschrieben, als „abweisend, unwissend und nur ihre medizinische Notdurft verrichtend“ [3].

Die Entlassungsdiagnose lautet: secundär fibröse Phthise bei Pneumoperitoneum und Phrenicusparese rechts. Empfohlen wird „die Fortsetzung eines kurmäßigen Lebens bei Durchführung einer Mittagsliegekur von 2-3 Stunden“.

Eine Reaktivierung ist in der Folgezeit nicht aufgetreten. Die Lungentuberkulose, der Darstellung von Thomas Bernhard folgend oft als die „Lebenskrankheit“ oder auch die „Todeskrankheit“ bezeichnet, hat vom Juni 1948 (Pleuritis exsudativa) bis zum Juli 1950 (Sputumkonversion) gedauert bzw. 21 / 2 Jahre, wenn man die kurmäßige Nachbehandlung in der Lungenheilstätte mitrechnet. Es hat sich um eine leichte Verlaufsform gehandelt.

Über fast zwei Jahrzehnte erfreute sich Thomas Bernhard dann einer guten Gesundheit und war auch körperlich leistungsfähig. In dem „versiegelten Tagebuch 1972“, in dem Hennetmair über fast tägliche Begegnungen minutiös berichtet, wird er als dynamisch und normal körperlich belastbar geschildert [9].

1967 erkrankt Thomas Bernhard an einer akuten Sarkoidose mit bihilärer Adenopathie. Die Diagnose wird in der Lungenklinik „Baumgartner Höhe“ bei Wien durch eine Mediastinoskopie gesichert. Bernhard berichtet in der Erzählung „Wittgensteins Neffe“, die zu seinen autobiografischen Schriften gerechnet wird, von Ärzten, „die mir den Hals aufschnitten, um aus meinem Brustkorb einen faustgroßen Tumor herausoperieren zu können. Als Folge meiner radikal an mir vorgenommenen Cortisonbehandlung entwickelte sich mein Mondgesicht, wie von den Ärzten gewünscht; während der Visite kommentierten sie dieses Mondgesicht auf ihre witzige Art, die selbst mich, der ich, nach ihrer eigenen Aussage, nur noch Wochen, im besten Falle Monate zu leben hatte, zum Lachen brachte“ [10]. Es ist kaum anzunehmen, dass ein Arzt eine solche Prognose gestellt hat. Offensichtlich hat der von Höller gelobte Realismus der Pathographie auch an dieser Stelle Grenzen. Sie mögen im laienhaften Verständnis des Patienten begründet sein oder im Bedürfnis des Autors, Krankheit und Todesnähe als gestalterisches Mittel einzusetzen.

Etwa seit 1980 machen sich Atembeschwerden bemerkbar, die allmählich zunehmen. Einem Arztbrief aus dem Krankenhaus Wels vom 21.12.1981 ist Folgendes zu entnehmen:

Thomas Bernhard ist weniger belastbar seit Sommer 1980 und seit Februar 1981 vermehrt kurzatmig; später traten nächtliche Atemnotanfälle auf, die sich nach Diuretika-Gabe besserten. Bei der Auskultation blasendes systolisches Geräusch mit p.max. über der Herzspitze. Im EKG intraventrikuläre Leitungsstörungen, mäßige ERBS. Echokardiografie: deutlich dilatierter linker
Ventrikel, erweiterter linker Vorhof. Thorax-Aufnahme: Herz nach links verbreitert.

Lungenfunktion vom 10.12.1981:

TLC 4,97 l (90\% Soll); VK 2,77 l (76\% Soll); FEV1 1.65 I (65\% Soll); FEV1 /VC=59\%; ITGV 3,551 (121\% Soll).TCO 82\%; TCO/VA 78\% des Sollwerts.

Ergänzend sei das Ergebnis eines pneumologischen Konsils vom Mai 1979 im Krankenhaus Wels mitgeteilt:

Thoraxaufnahme: unscharf begrenzte Verbeiterung und Verdichtung der Hili. Strukturverdichtung rechts parahilär. Pleuraschwarte im Bereich des rechten Mittel-Oberfeldes. Pleuraadhäsionen basal.

Beurteilung: Leicht- bis mittelgradige restriktive Ventilationsstörung mit anamnestisch und klinisch fallweiser zusätzlicher Obstruktion. Aufgrund der Vorgeschichte, der Röntgenbilder und der Lungenfunktion ist eine Lungensarkoidose mit beginnender Fibrosierung anzunehmen.

Obwohl die Diagnose z. B. Lungenfibrose bei M. Boeck in Berichten auftaucht, sind die radiologischen Befunde und die Lungenfunktionswerte nicht im Sinne einer Sarkoidose im Stadium III zu interpretieren. Die Symptome der Herzinsuffizienz - allmählich zunehmende Belastungsdyspnoe („damals hatte ich die Kraft, zu gehen und zu singen, dachte ich auf dem Ohrensessel, heute habe ich nicht mehr die Kraft, zu gehen und zu sprechen, das ist der Unterschied“) [11] und Episoden nächtlicher Atemnot standen im Vordergrund. Wahrscheinlich war die der Herzinsuffizienz zugrundeliegende dilatative Cardiomyopathie die Folge einer Herzbeteiligung bei Sarkoidose. Der Exitus trat am 12. Februar 1989 unter den Zeichen des therapieresistenten Linksherzversagens ein (persönliche Mitteilung Dr. Peter Fabian). Eine Autopsie wurde nicht durchgeführt.

\section{Thomas Bernhard - ein schwieriger Patient?}

Das Milieu - „die entsetzlichen Zustände einer öffentlichen Lungenheilstätte“- wird beeindruckend geschildert: „, In ihren schäbigen Nachkriegsschlafröcken, abgetretenen Filzpantoffeln, schmutzigen Nachthemdenkragen zogen die Patienten, die Fiebertafeln unter ihre Arme geklemmt, hintereinander...ihr Ziel war die Liegehalle gewesen, eine halbverfallene Holzveranda im Freien...Im Vorbeigehen schraubten diese zweifellos endgültig aus der Menschengesellschaft Ausgestoßenen widerwärtig, armselig und wie in einem heiligen Stolz verletzt, ihre braunen Glasspuckflaschen auf und spuckten hinein, mit einer perfiden Feierlichkeit holten sie hier überall schamlos und in einer nur ihnen eigenen raffinierten Kunst das Sputum aus ihren zerfressenen Lungenflügeln und spuckten es in die Spuckflaschen ... “ [5].Thomas Bernhard fühlt sich dieser Patientengruppe nicht zugehörig, denn er hat zunächst kein Sputum. Nach fünf Wochen gelingt es ihm, Sputum abzuliefern, und diese Probe ist angeblich positiv. Er reagiert mit Resignation: „Warum sollte gerade ich...glauben oder auch nur einen Augenblick lang in Anspruch nehmen dürfen...davonzukommen, wo Millionen ganz einfach nicht davongekommen waren? Ich hatte jetzt, so mein Gedanke, den direkten Weg durch die Hölle und in den Tod zu gehen....Ich hatte mich damit abgefunden, ich fügte mich.“ Am gleichen Tag aber stellt sich heraus, dass die Probe im Labor verwechselt worden war. Die richtige Sputumprobe und weitere Kontrollen waren negativ. Der gerade noch zu Tode Erschrockene atmet nicht auf, wie man erwarten würde, sondern gibt sich kämpferisch. Schon eine Seite weiter lesen wir: „Ich lehnte mich heftiger denn je auf gegen 
Grafenhof und seine Gesetze, gegen die Unausweichlichkeit! Ich hatte meinen Standpunkt wieder am radikalsten geändert ... ich forderte von mir die höchste Aufmerksamkeit, vor allem eine noch schärfere Arztkontrolle ... oberflächlich fügte ich mich der Hausordnung, der medizinischen Gewalt, unter dieser Oberfläche bekämpfte ich sie... ich bestimmte, wie viel Streptomycin ich zu bekommen hatte, nicht die Ärzte, aber ich ließ sie in dem Glauben, dass sie es bestimmten, alle meine Peiniger ließ ich in dem Glauben, dass sie es bestimmten, was zu geschehen habe, während doch von jetzt an nur geschah, was ich bestimmte..." Und folgerichtig entlässt er sich auch selbst: „Ich wollte gehen, also ging ich, ich war es gewesen, der meine Entlassung bestimmte, obwohl die Ärzte dann das Gefühl hatten, sie hätten mich entlassen.“

Ist der zuständige Stationsarzt zu bedauern? Nicht unbedingt, denn er bemerkt die Aufsässigkeit seines Patienten gar nicht. „Es ist dieselbe Verfahrensweise, durch die sich jemand, der sich für Napoleon hält, mit der Welt arrangiert: er verschweigt einfach, dass er Napoleon ist. Er hält die Wahrheit unter der Oberfläche, unter Verschluss und lässt die anderen glauben, er sei gar nicht Napoleon" [12].

Und das Pendel der Verantwortung für den Krankheitsverlauf schwingt denn auch zurück, als zwei Monate später bei einer Thorax-Kontrolle ein Infiltrat festgestellt wird. Nun klagt er die Ärzte an, denn „sie mussten wissen, dass sie mir nicht aus Unschuld, sondern aus Schuld eine Fehldiagnose gestellt hatten."

\section{Die autobiografischen Erzählungen - eine Pathografie?}

$\nabla$

Die Texte überzeugen auf Anhieb durch ihre sprachliche Brillianz und zahlreiche Details, die einen Tatsachenbericht und medizinische Sachkenntnis suggerieren. Bei genauem Lesen stellt man allerdings fest, dass es naiv wäre, von einer Pathografie zu sprechen und der Darstellung des Autors bedenkenlos zu folgen. Als Ergebnis einer sorgfältigen textkritischen Analyse stellt Maier fest: „Bernhards Autobiographien sind rhetorisch. Situationen oder Sachverhalte werden als pathetisierende Effekte verwendet und geraten dadurch in Widerspruch zu anderen Textpassagen. Das geht bis in die Mikroebene einzelner Sätze. Der Effekt ist wichtiger als der Wahrheitswille, letzterer ist vielmehr nirgendwo erkennbar...Am Ende erstarrt Bernhards Text-Ich in einer Allmachtsphantasie, die gezeichnet ist durch eine Realitätsverdrehung, wie sie sonst in Autobiographien noch nicht zu sehen war" [12].
Für mich bleibt als Fazit dieser Nachforschungen, dass die Übergänge von der Realität zur Fiktion fließend sind. Thomas Bernhard hat Wochen und Monate in Krankenhäusern und Heilstätten verbracht, war den geschilderten medizinischen Prozeduren tatsächlich ausgesetzt, hat das Elend der Kranken und die Hilflosigkeit der Ärzte in der Ära vor einer wirksamen Pharmakotherapie der Tuberkulose kennengelernt. Wir verdanken ihm eine authentische, plastische Schilderung des Milieus, die an den Zauberberg denken lässt. Aus seinen Erfahrungen als Patient hat er Textmaterial gewonnen und phantasievoll gestaltet. Es spricht für die suggestive Kraft seiner Darstellung, für dieses beispielsweise auch in der Erzählung Holzfällen erkennbare „teils kunstvolle, teils bewusst offensichtliche Spiel mit autobiographischen Elementen“ [13], dass die leichte Verlaufsform einer im Adoleszentenalter abgelaufenen Tuberkulose als die Lebenskrankheit oder Todeskrankheit wahrgenommen wurde.

\section{Interessenkonflikte}

$\nabla$

Der Autor gibt an, dass kein Interessenkonflikt besteht.

\section{Literatur}

1 Roth P. Eigene und fremde Bücher, wiedergelesen. Reinbek bei Hamburg: Rowohlt Taschenbuch Verlag, 2009: 225

2 Mittermayer M. Thomas Bernhard und die Lungenkrankheit. Beobachtungen an seinen autobiographischen Schriften. Atemw-Lungenkrh 2000; 26: $158-166$

3 Bernhard T. Der Atem. Eine Entscheidung. München: Deutscher Taschenbuchverlag, 2001

4 Bernhard T. Die Ursache. Eine Andeutung. München: Deutscher Taschenbuchverlag, 2001

5 Bernhard T. Die Kälte. Eine Isolation. München: Deutscher Taschenbuchverlag, 2001

6 Bernhard T. Ein Kind. München: Deutscher Taschenbuchverlag, 2001

7 Huguet L. Chronologie. Johannes Freumbichler/Thomas Bernhard. Weitra: Bibliothek der Provinz, 1995

8 Höller $H$. Thomas Bernhard. rowohlts monographien, 6. Aufl. Reinbek bei Hamburg: Rowohlt Taschenbuch Verlag, 2000

9 Hennetmair I. Ein Jahr mit Thomas Bernhard. Das versiegelte Tagebuch. 1972. Salzburg und Wien: Btb, 2003

10 Bernhard T. Wittgensteins Neffe. Eine Freundschaft. Frankfurt a.M: Suhrkamp, 1982

11 Bernhard T. Holzfällen. Eine Erregung. Frankfurt a. M: Suhrkamp, 1984

12 Maier A. Die Verführung. Thomas Bernhards Prosa. Wallstein: Göttingen, 2004

13 Kehlmann D. Der kühle Lobbyist in der Maske des manischen Bezichtigers. FAZ Oktober 2007, Nr. 235 